\title{
Impulse signals classification using one dimensional convolutional neural network
}

\author{
Mikhail Olkhovskiy, Eva Müllerová, Petr Martínek ${ }^{1}$
}

\begin{abstract}
The main purpose of this work is to propose a modern one-dimensional convolutional neural network (1 D CNN) configurations for distinguishing separate PD impulses from different types of PD sources while the parameters of these sources are changed. Three PD sources were built for signal generation: corona discharge, discharge in a void, and surface discharge. The reason for using separate PD impulses for classification is to develop a universal tool with the ability to recognize an insulation defects by analysing very few events in the insulation in a short range of time. Additionally, we found the optimal sample rates for the data acquisition for these network configurations. The necessity of signal filtering was also tested. The following configurations of a neural network were proposed: configuration for classification raw PD impulses; configuration for classification of PD impulses represented by power spectral density, for both filtered and unfiltered variants.

Keywords: convolutional neural networks, one-dimensional convolutional neural network, partial discharge, signal analysis
\end{abstract}

\section{Introduction}

For analyzing PD signals caused by defects in high voltage equipment, the artificial neural networks (ANN) are being widely used. During the last years, conventional ANNs configurations for PD recognition and classification were proposed by many authors. Papers are devoted to $\mathrm{PD}$ phenomenon in a wide range of equipment such as gas insulated switchgears [1], power transformers [2], cables [3], and insulators [4]. Some researchers use laboratory setups to model PD activity for determining the optimal neural network type and its configuration [5].

Generally, PD signal processing in the case of conventional ANN applications, consists of the next steps: data acquisition, signal denoising, PD feature extraction, and PD classification, Fig. 1(a). Denoising is an essential procedure for conventional PD measurements because external noise interference can significantly degrade the measurement sensitivity. Feature extraction is a following necessary part of data processing when conventional neural networks are used. The aim of a feature extraction is to obtain the relevant features of a PD signal to determine the characteristics correlated with a specific insulating defect. Statistical parameters, such as: skewness, kurtosis, mean, variance, and cross-correlation factor, are commonly used for feature extraction. Moreover, frequency and time features, fractal features, cross wavelet spectrum, two-pass split window scheme, autocorrelation technics, and even chaos theory can be applied to a feature generation, the overview of these technics can be found in work [6].

Neural networks which consist of more than two hidden layers, are commonly called deep neural networks. If some of the hidden layers are represented by convolutional filters, then such a network is called Convolutional Neural Network (CNN). The most significant ad-

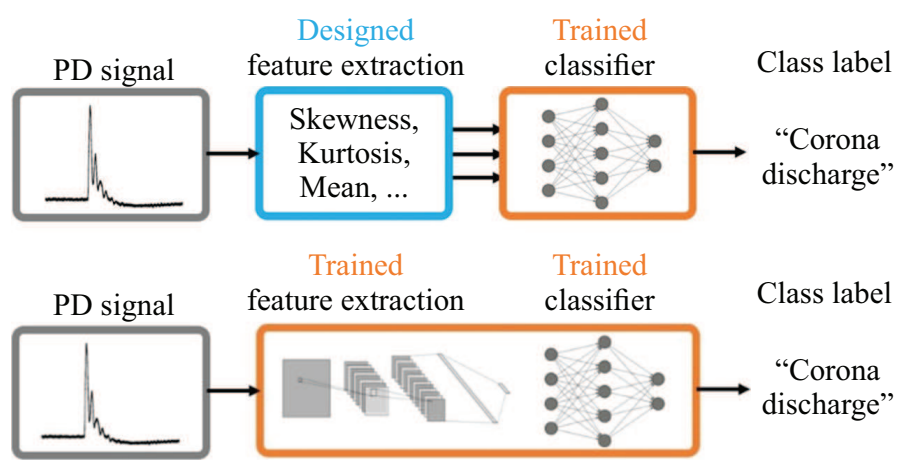

Fig. 1. Comparing the conventional neural network classification using: (a) - manually designed features, and (b) - classification with deep-learning structures, [7]

Department of Electrical Power Engineering and Environmental Engineering, Faculty of Electrical Engineering, University of West Bohemia, Pilsen 30100, Czech Republic, mikhail@fel.zcu.cz

DOI:10.2478/jee-2020-0054, Print (till 2015) ISSN 1335-3632, On-line ISSN 1339-309X

(C) This is an open access article licensed under the Creative Commons Attribution-NonCommercial-NoDerivs License (http: //creativecommons.org/licenses/by-nc-nd/3.0/). 


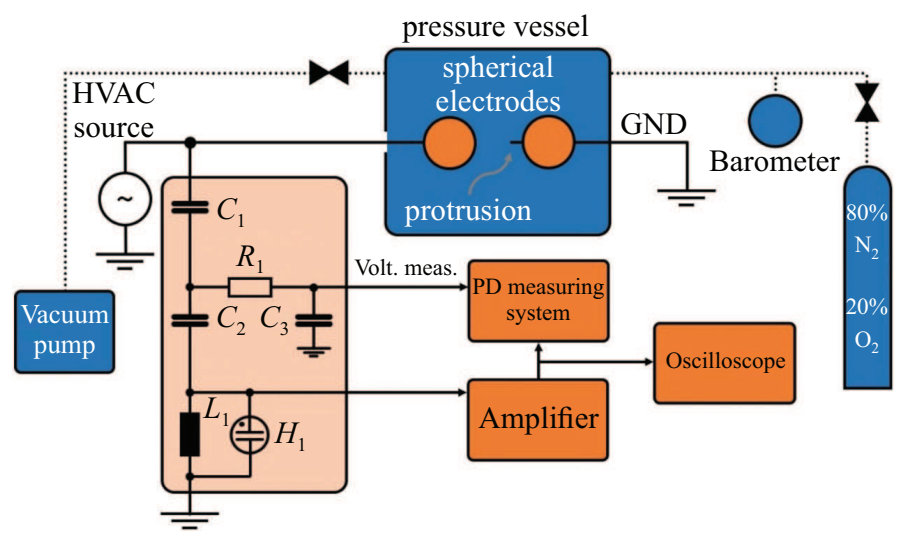

Fig. 2. Schematic representation of the experimental setup for the case of corona discharge

vantage a CNNs offers is that they can combine feature extraction and classification tasks in one structure. CNNbased networks learn to extract features from a signal, these features are guaranteed to be relevant to the specific insulating defect. Therefore, there is no necessity for human-constructed features as is in conventional ANNs. It is graphically expressed in Fig. 1(b).

CNNs were initially designed to process data represented in the form of $2 \mathrm{D}$ arrays since it was necessary to classify images. It is the reason for using phase-resolved partial discharge (PRPD) diagram, phase-resolved pulse sequence (PRPS) diagram, and normalized differenced (NoDi) pattern for PD signal classification by $2 \mathrm{D}$ CNNs. PRPD diagram is a $f-q-n$ histogram in which $f$ is a phase position, $q$ is a charge, and $n$ is a density [8]. PRPS diagram uses the time difference between PD events. NoDi pattern was developed for direct current system diagnosis and uses the time difference between PD impulses also. Another way to transform $1 \mathrm{D}$ data into $2 \mathrm{D}$ form is the application of a Fourier transformation, resulting in frequency-time representation $[9,10]$. In work [11], the shapes of PD signals in time domain and their spectra are used to overcome the interference problem to detect different types of defects in high voltage power cables.

In the case of $2 \mathrm{D}$ representation, a conclusion regarding the nature of a PD signal is made based on analyzing the high level features of the signal (skewness, kurtosis, etc.). It means that information about the shape of the individual PD impulses is completely lost. Method proposed in this work allows us to use information hidden in the shape of the individual PD impulse and, at the same time, a possibility for further analyzing of an information about the time of pulse occurrence remains. Moreover, in this case, we use a raw PD signal in the form of one-dimensional tensor, which allows us to apply modern mathematical algorithms in the form of $1 \mathrm{D}$ CNN to process raw PD impulses with minimal preprocessing, ie . without transformation to a $2 \mathrm{D}$ representation. Such steps have also been made by some researchers, for example, work [10] proposed a $1 \mathrm{D}$ CNN to classify 3 different types of PDs in power transformers by using signal from acoustic emission sensors.
In our work, we concerned a network configuration for distinguishing not only impulses from a different source of PD signal, but also for distinguishing impulses from the same type of PD source with a slightly different source configuration, such as pressure, electrode distances, void diameter and others, described in the next chapters.Due to using separate PD impulses for analysis, proposed $1 \mathrm{D}$ CNN configuration can be also applied for distinguishing multiple discharge sources. The only difficulty that can arise is overlapping two or more impulses from two or more sources or from the same source of PD signal. The probability of these events and the level of affecting of algorithm performance will be determined in the farther experiments. Another reason for using separate PD impulses is to obtain a universal tools for analizing the signal also in DC equipment, where the phase angle is absent.

Table 1. The combinations of the parameters and corresponding class names for corona discharge

\begin{tabular}{lccc}
\hline $\begin{array}{l}\text { Electrode } \\
\text { distance } \\
(\mathrm{mm})\end{array}$ & $\begin{array}{c}\text { Pressure } \\
(\mathrm{kPa})\end{array}$ & $\begin{array}{c}\text { Protrusion } \\
\text { length } \\
(\mathrm{mm})\end{array}$ & $\begin{array}{c}\text { Class } \\
\text { name }\end{array}$ \\
\hline 30 & 300 & 5 & $\mathrm{n} 0$ \\
30 & 300 & 2 & $\mathrm{n} 1$ \\
30 & 250 & 2 & $\mathrm{n} 2$ \\
30 & 250 & 5 & $\mathrm{n} 3$ \\
40 & 300 & 5 & $\mathrm{n} 4$ \\
40 & 300 & 2 & $\mathrm{n} 5$ \\
40 & 250 & 2 & $\mathrm{n} 6$ \\
40 & 250 & 5 & $\mathrm{n} 7$ \\
\hline
\end{tabular}

\section{Experimental setup}

To test the algorithm, three types of PD were chosen: corona discharge, discharge in a void, and surface discharge. Setup for the generation of a corona discharge consists of two spherical electrodes with a diameter of 15 $\mathrm{cm}$, placed into the metallic vessel (Fig. 2). One of the 
Table 2. The combinations of the parameters and corresponding class names for internal discharge

\begin{tabular}{lccc}
\hline $\begin{array}{l}\text { Electrode } \\
\text { distance } \\
(\mathrm{mm})\end{array}$ & $\begin{array}{c}\text { Void } \\
\text { diameter } \\
(\mathrm{mm})\end{array}$ & $\begin{array}{c}\text { Void } \\
\text { length } \\
(\mathrm{mm})\end{array}$ & $\begin{array}{c}\text { Class } \\
\text { name }\end{array}$ \\
\hline 4 & 1.5 & 1.5 & $\mathrm{n} 8$ \\
4 & 2.5 & 2.5 & $\mathrm{n} 9$ \\
\hline
\end{tabular}
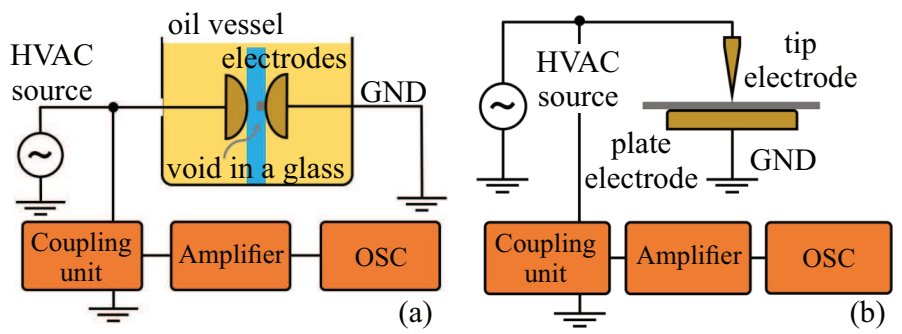

Fig. 3. Schematic representation of the experimental setup: (a) - volume discharge, and (b) - surface discharge.

electrodes is connected to a high voltage source, represented by a laboratory $50 \mathrm{~Hz}$ PD-free transformer. Another electrode is grounded. Grounded electrode has an artificial protrusion in the form of a tip with a radius of curvature $0,5 \mathrm{~mm}$. Length of the artificial protrusion can be set in the range from 0 to $10 \mathrm{~mm}$. Before filling with synthetic air, the vessel was evacuated by a vacuum pump to reach a medium vacuum inside. In this experiment, impulses from corona discharge were obtained for two levels of pressure: $250 \mathrm{kPa}$ and $300 \mathrm{kPa}$. Second parameter was distance between the spherical electrodes, it was $30 \mathrm{~mm}$ and $40 \mathrm{~mm}$. Third parameter was a protrusion length: 2 $\mathrm{mm}$ and $5 \mathrm{~mm}$. Combining these parameters, eight separate classes of corona discharge, named from $\mathrm{n} 0$ to $\mathrm{n} 7$, were obtained and recorded. These combinations are represented in Tab 1.

In Fig. 3(a), the setup for obtaining the signal from the internal discharge is shown. It includes a container with two electrodes filled with insulation oil. Between the electrodes, a plate made of insulation material was placed containing an artificial void with a dimension represented in Table 2. There are two variants: the first of them had the void with $1,5 \mathrm{~mm}$ diameter and 1,5 $\mathrm{mm}$ length, and the second one had the void with $2,5 \mathrm{~mm}$ diameter and 2,5 mm length.

Next type of PD was a surface discharge, Fig. 3(b). The setup for this type was arranged in such way that the grounded electrode was placed under the insulating plate, while the energized upper tip electrode is in touch with the insulating plate. The thickness of the insulating material is $2,6 \mathrm{~mm}$.

Commercial PD analyzer was used to control the presence of partial discharge and a type of partial discharge.
Table 3. The combinations of the parameters and corresponding class names for surface discharge

\begin{tabular}{lccc}
\hline $\begin{array}{l}\text { Insulation } \\
\text { sheet } \\
\text { thickness }\end{array}$ & $\begin{array}{l}\text { Grounded } \\
\text { electrode } \\
\text { diameter }\end{array}$ & $\begin{array}{c}\text { Tip } \\
\text { electrode } \\
\text { curvature }\end{array}$ & $\begin{array}{c}\text { Class } \\
\text { name }\end{array}$ \\
\hline $2.6 \mathrm{~mm}$ & $75 \mathrm{~mm}$ & $0.5 \mathrm{~mm}$ & $\mathrm{n} 10$ \\
\hline
\end{tabular}

\section{Data acquisition and processing}

As an input for ANN, often, preprocessed PD signal is used. Therefore, phase-resolved partial discharge (PRPD) diagram and phase-resolved pulse sequence (PRPS) diagram are usually applied in the case of alternating current $(\mathrm{AC})$, and a normalized differenced (NoDi) pattern for the case of direct current (DC) [15]. The main purpose of our work is to propose and test an algorithm for processing raw $\mathrm{PD}$ impulses, it is without the construction of a PD diagram. The reason is to develop a universal tools with the ability to recognize an insulation defects by analyzing very few $\mathrm{PD}$ impulses in a short range of time.

To provide separate PD impulses to ANNs input, two possibilities exists. The first theme is to use an efficient algorithm for detecting and extracting impulses from time series data [16]. Then, the extracted PD impulses can be pass to the ANNs input. This method is suitable for processing PD signals obtained in the field condition. The second one is to use oscilloscope with a predefined time window and predefined trigger level, in this way we obtain PD impulses already prepared for ANN learning, validation and testing. This method is appropriate for laboratory conditions. Because the primary aim of our work is to propose a $1 \mathrm{D}$ CNN configuration for distinguishing separate $\mathrm{PD}$ impulses, we decided to use the second method, consisting in the use of already separated PD impulses.

Data acquisition was performed by means of a capacitive-inductive coupling unit, which is normally used for partial discharge measuring, according to testing standards. Coupling unit was connected to a digital oscilloscope through an amplifier with bandwidth $40 \mathrm{kHz} 20$ $\mathrm{MHz}$. Time window during signal acquisition was set to $5000 \mathrm{~ns}(500 \mathrm{~ns} /$ div). Every digitized PD signal record contains 50000 significant sampled values (10 GS/s). Captured PD impulses were stored on the oscilloscope storage 
Table 4. Corona discharge recorded PD impulses: class/electrode distance/pressure

Protrusion length $2 \mathrm{~mm}$
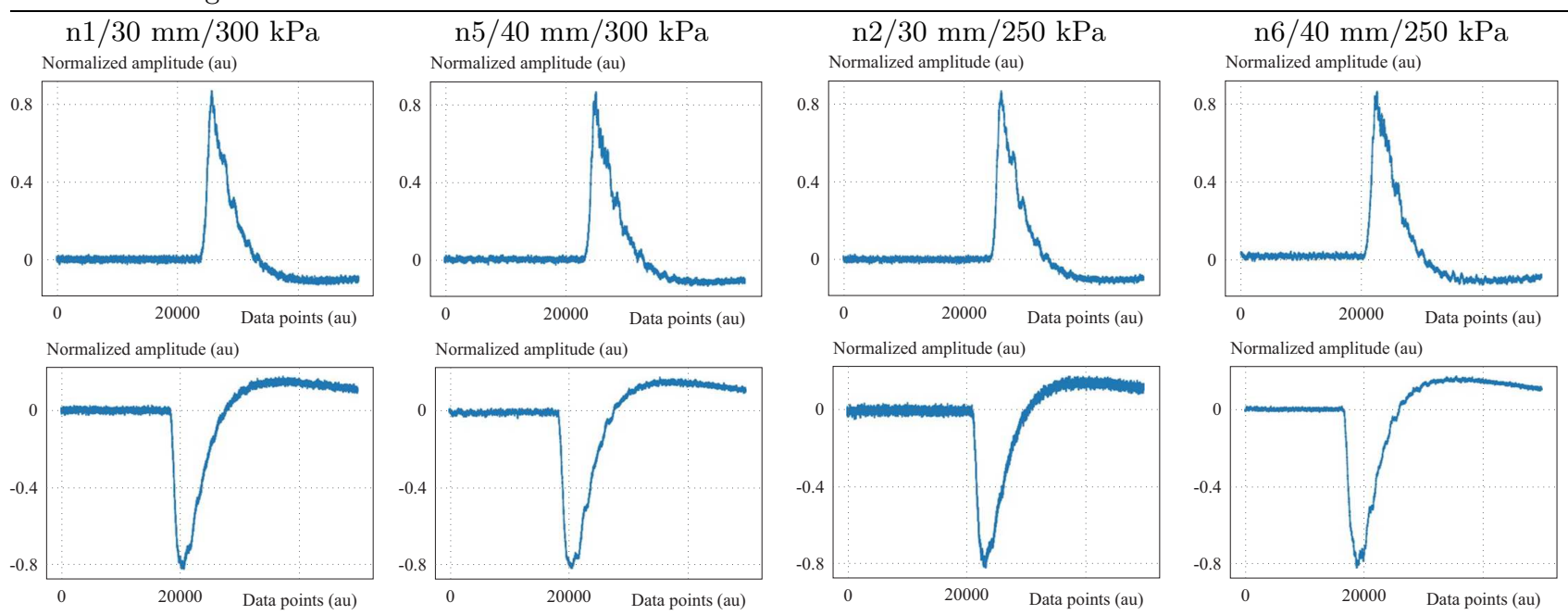

Normalized amplitude (au)
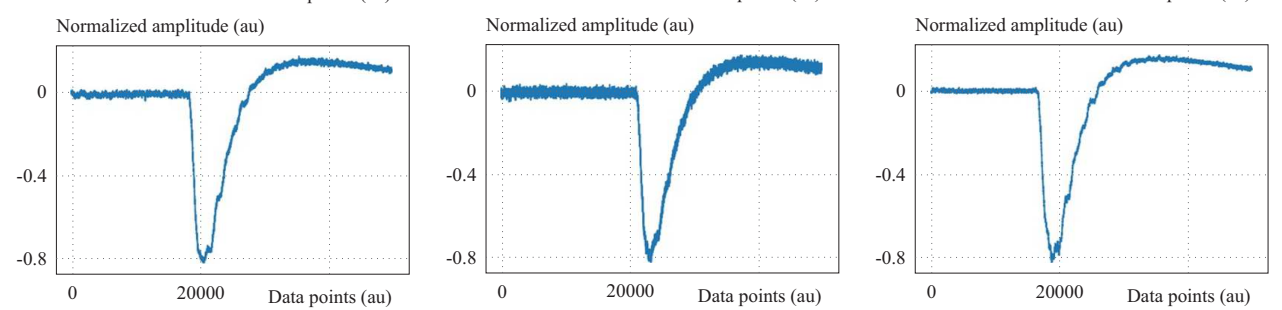

Protrusion length $5 \mathrm{~mm}$
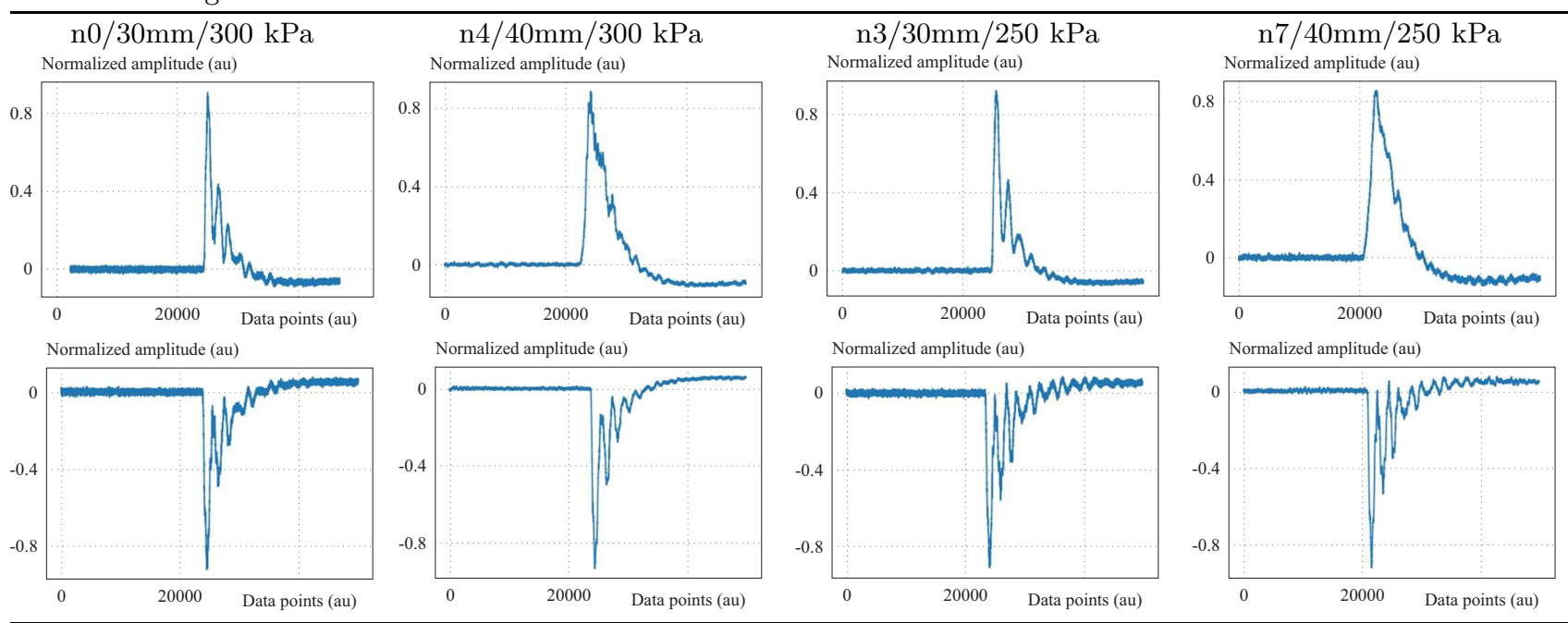

Table 5. Recorded PD impulses for discharge in a void (different diameters in $\mathrm{mm}$ ) and surface discharge

Void dischage

Surface discharge
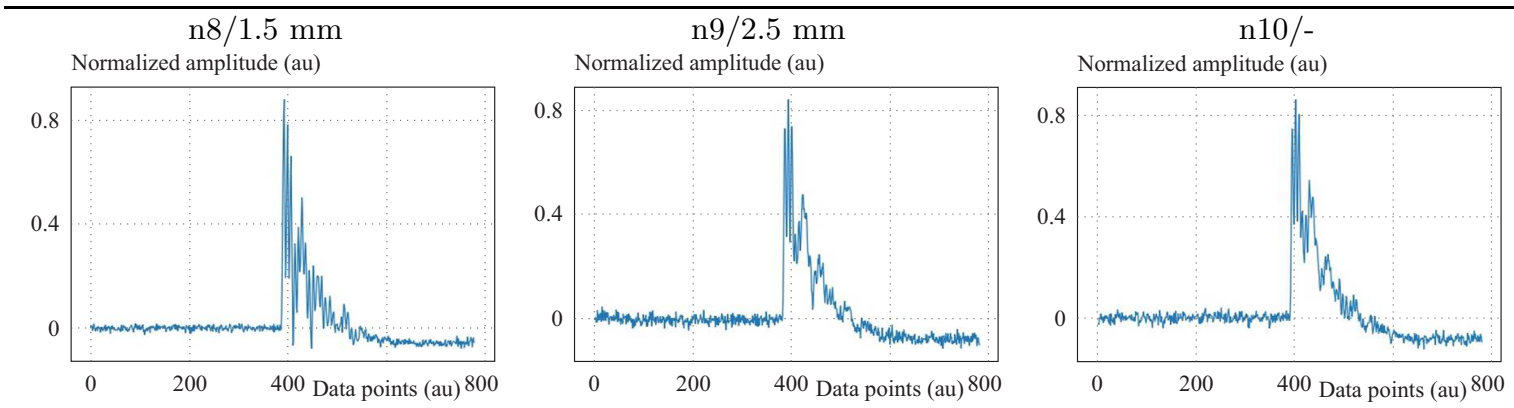

for further processing. All data processing was done by using Python programming language and its toolkits.

Each class from n0 to n10 contains 200 records, in that, classes n0-n7 contain 100 records with positive polarity impulses and 100 records with negative polarity impulses.
To keep versatility and reproducibility, the amplitudes of $\mathrm{PD}$ impulses were normalized in a range from 0 to 1. Examples of normalized impulses, corresponding to each class, are shown in the tables below. 
Table 6. Time-per-step parameter during learning process for different sample rate of the signal

\begin{tabular}{lccccc}
\hline $\begin{array}{l}\text { Processing } \\
\text { abbreviation }\end{array}$ & $\begin{array}{c}\text { Samples } \\
\text { per window }\end{array}$ & $\begin{array}{c}\text { Window } \\
\text { length }\end{array}$ & $\begin{array}{c}\text { Sampling } \\
\text { factor }\end{array}$ & $\begin{array}{c}\text { Sample rate } \\
\text { Sample }\end{array}$ & $\begin{array}{c}\text { Learning: } \\
\text { time/step }(\mu \mathrm{s})\end{array}$ \\
\hline W0-S50000 & 50000 & 0 & & $10 \mathrm{GS} / \mathrm{s}$ & 3000 \\
W81-S50000 & 50000 & 81 & & $10 \mathrm{GS} / \mathrm{s}$ & 3000 \\
W81-S25000 & 25000 & 81 & 2 & $5 \mathrm{GS} / \mathrm{s}$ & 1000 \\
W81-S12500 & 12500 & 81 & 4 & $2.5 \mathrm{GS} / \mathrm{s}$ & 600 \\
W81-S6250 & 6250 & 81 & 8 & $1.25 \mathrm{GS} / \mathrm{s}$ & 307 \\
W81-S3125 & 3125 & 81 & 16 & $625 \mathrm{MS} / \mathrm{s}$ & 170 \\
W81-S1562 & 1562 & 81 & 32 & $312 \mathrm{MS} / \mathrm{s}$ & 100 \\
W81-S781 & 781 & 81 & 64 & $156 \mathrm{MS} / \mathrm{s}$ & 70 \\
W0-S781 & 781 & 0 & 64 & $156 \mathrm{MS} / \mathrm{s}$ & 70 \\
W81-S390 & 390 & 81 & 128 & $78 \mathrm{MS} / \mathrm{s}$ & 56 \\
W81-S195 & 195 & 81 & 256 & $39 \mathrm{MS} / \mathrm{s}$ & 42 \\
W81-S97 & 97 & 81 & 512 & $19 \mathrm{MS} / \mathrm{s}$ & 42 \\
W81-S48 & 48 & 81 & 1024 & $9.6 \mathrm{MS} / \mathrm{s}$ & 42 \\
\hline
\end{tabular}

For applying the classification algorithm, 200 records within every class were divided in the following way: 140 records were used as a training data set (70 negative and 70 positive, for the case of classes n0-n7), 40 records were used as a validation data set (20 negative and 20 positive, for the case of classes n0-n7) and 20 records as a testing data set (10 negative and 10 positive, for the case of classes n0-n7).

Thus, to determine the difference in efficiency of the neural network signal processing, a moving average function was applied to the raw signal to suppress a background noise. From Fig. 4, it is apparent that a function window with length parameters 51 and 81 effectively suppresses the background noise and at the same time the details of the useful signal remain intact. It seems reasonable to use a function window length equal 81 for this experiment. Similar results can be obtained by application of a low pass Butterworth filter with cut-off frequency 120-150 MHz.

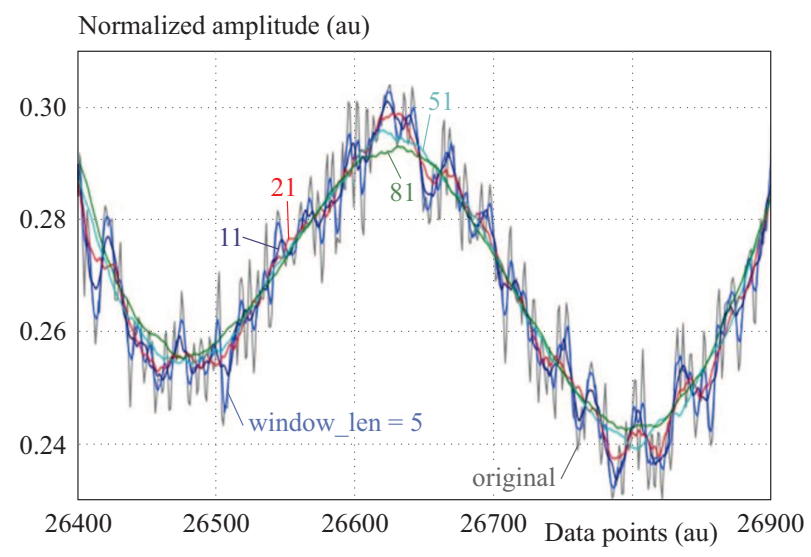

Fig. 4. Detail of the useful signal with different moving average function window length applied
To simulate the different sample rates of the signal acquisition equipment, the original signal was downsampled by different downsampling factors and, as a result, a set of variants, presented in Tab. 4, was obtained. Using a signal with low sampling rate helps to reduce the data volume and save the computational resources of the system. Consequently, downsampling allows to increase training and classification speed by using a simpler neural network structure. Such improvement in a learning speed is visible from time-per-step values (Tab. 6), obtained during the learning process. These time-per-step values are relevant for the computing system based on the graphical card GeForce GTX $1650(1,68 \mathrm{GHz})$ with 4 GB DDR5 $(8 \mathrm{GHz})$. For convenience, every version of signal processing corresponds to the relevant abbreviation. Where $\mathrm{W}$ indicates the window length of the smoothing average function, $\mathrm{S}$ is the number of samples contained in one $\mathrm{PD}$ record. Since it is planned to use cost-effective solution for PD signal acquisition for further experiments, variants of data processing, named W0-S781 and W81-S781, were chosen for using in the classification task. Configurations W0-S390, W81-S390, W0-S195, W81-S195 can be used for the classification of PD signals, but the classification capability for classes number 1 and number 5 significantly decreases in this case. Configurations W0S97, W81-S97, W0-S48, W81-S48 are not useful due to signal aliasing occurrence.

\section{Network configurations and results classification}

As stated above, one-dimensional convolutional neural network (1 D CNN) is considered to have great capability for $1 \mathrm{D}$ signal processing. According to this, the following structure, shown in Fig. 5, was proposed for data processing in this experiment for data configuration W0-S781. 


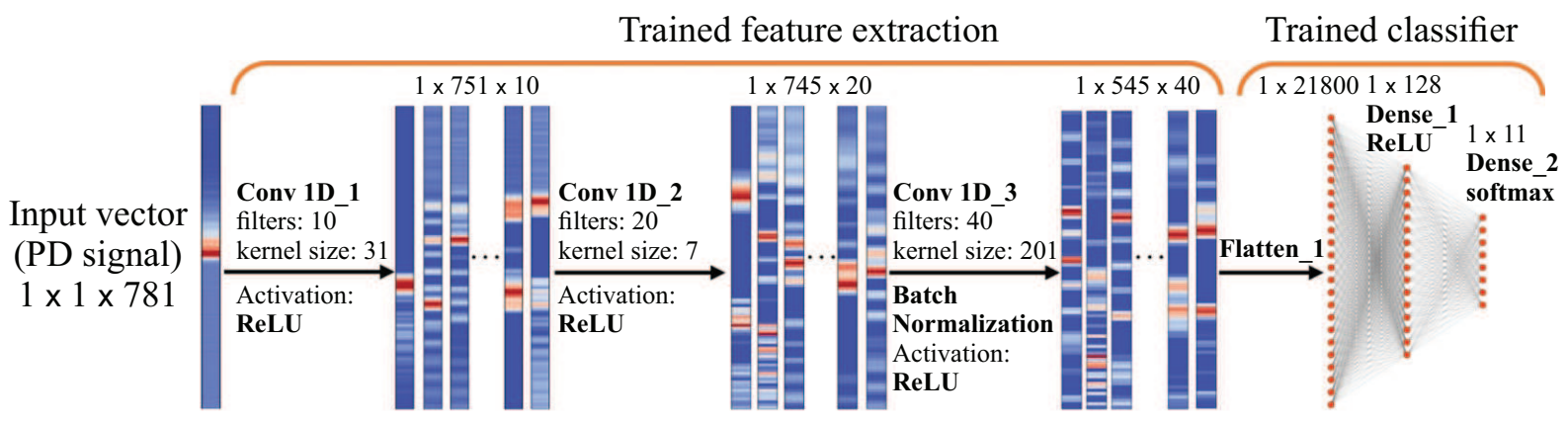

Fig. 5. 1 D CNN configuration for W0-S781 (Optimizer: Adam. batch size 170, epochs 80, loss function categorical cross entropy, metrics categorical accuracy )

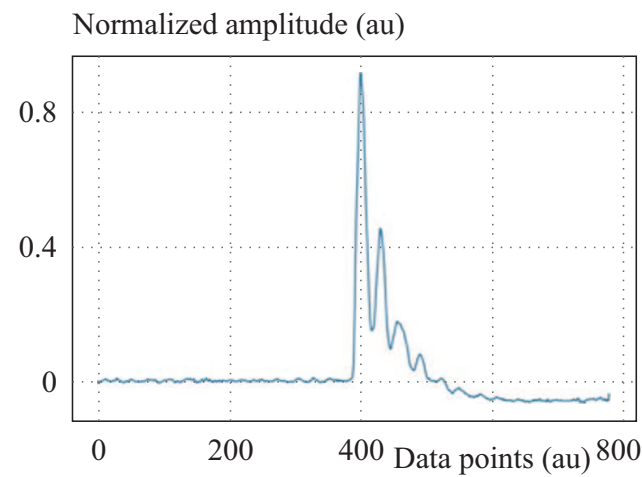

PSD normalized (au)

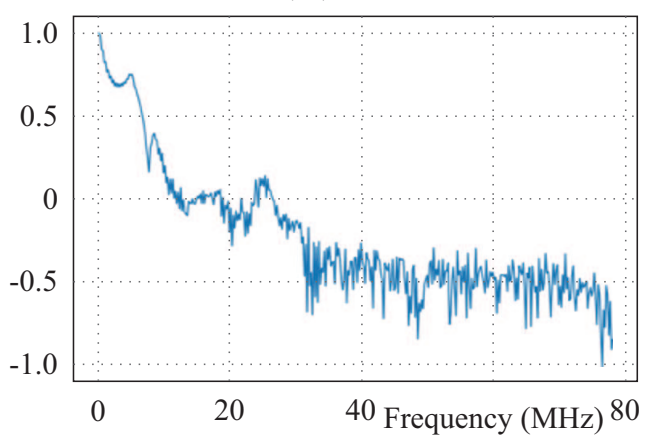

Normalized amplitude (au)

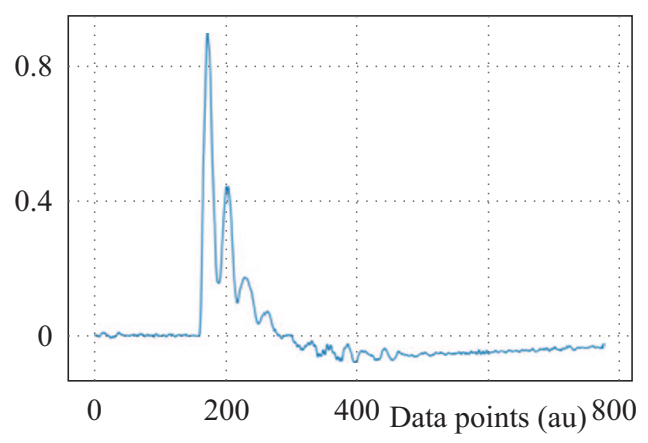

PSD normalized (au)

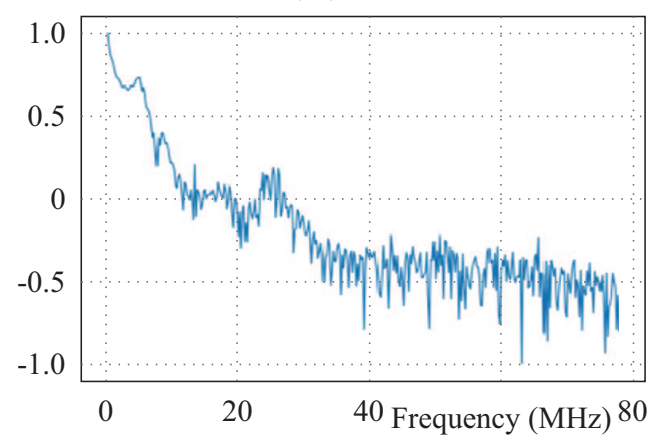

Fig. 6. (a) - original PD signal, (b) - PSD for original PD signal, (c) - time-shifted PD signal, (d) - PSD for time-shifted PD signal

As a part of data preparation, firstly, time-amplitude data values of the PD signal were extracted from TRC file (which is the oscilloscope file extension format), then they are placed in the $2 \mathrm{D}$ tensor with a size $M \times K$, where $M$ is depending on the number of training, validation or testing samples, respectively. $K$ is the number of sampled data points. Thus, for the configuration W0-S781, the parameter $K=781$, and for the training case we have the tensor with a size $140 \times 781$, for validation, with a size $40 \times 781$, for testing with a size $20 \times 781$. Simultaneously, $1 \mathrm{D}$ tensors consisting of information about classes which corresponds to the relative PD signal sample and with a size of $M \times 1$ are generated.

Before placing the data into the $2 \mathrm{D}$ tensor, every amplitude vector passed through a normalization process, where the amplitude volumes were normalized in a range from 0 to 1 . After normalization, the smoothing average function (low pass filtering) was applied. Afterwards, a downsampling procedure was employed to simulate different sampling frequency and its influence. Subsequently, the operation of concatenation was applied and the following tensors are obtained by using in the network training and validation process: for training it is a $1540 \times 781$ $2 \mathrm{D}$ tensor with corresponding class number in the form of $1540 \times 1$ vector; for validation it is a $440 \times 7812 \mathrm{D}$ tensor with corresponding class number in the form of $440 \times 1$ vector; for testing it is $220 \times 7812 \mathrm{D}$ tensor with corresponding class numbers in form of $220 \times 1$ vector.

Network construction was realized by high-level neural network API Keras [12]. The network has an input layer, 
Table 7. Proposed network structures for using PSD as an input for the 1D CNN; parameters: filters/kernel-size, $N$-neurons

\begin{tabular}{|c|c|c|c|}
\hline \multirow[t]{2}{*}{ Layer } & \multicolumn{2}{|c|}{ Parameters } & \multirow[t]{2}{*}{ Activation } \\
\hline & W81-S781-PSD & W0-S781-PSD & \\
\hline Conv1D & $10 / 31$ & $5 / 3$ & ReLU \\
\hline Conv1D & $20 / 7$ & $10 / 7$ & ReLU \\
\hline Dropout & 0.5 & 0.5 & \\
\hline Conv1D & $40 / 3$ & $20 / 31$ & ReLU \\
\hline \multicolumn{4}{|c|}{ Batch normalization } \\
\hline Dropout & 0.5 & 0.5 & \\
\hline & Flatten & & \\
\hline Dense & $N: 128$ & $N: 128$ & ReLU \\
\hline \multicolumn{4}{|c|}{ Batch normalization } \\
\hline Dropout & 0.5 & 0.5 & \\
\hline Dense & $N: 11$ & $N: 11$ & softmax \\
\hline
\end{tabular}

hidden layers in the form of convolutional and dropout layers in different combinations, and conventional fully connected layers at the output (Fig. 5). General rule is that with the number of hidden layers increasing, the capabilities of CNN increases significantly, and more samples are needed to avoid overfitting. Overfitting is a situation when a network describes the training data set well but does not have enough predictive ability for new data. Pooling layers are usually inserted between convolutional ones. They reduce the spatial data size and help to control an overfitting. It turned out that the pooling layer did not work properly in our case and, therefore, do not include it in the network structure. Furthermore, batch normalization was employed to speed up the training process. The Softmax function is applied as an activation function of the output layer. This function returns the probability distribution over mutually exclusive output classes for multiclass classification problems [13].

We have also tested behaviour of the proposed network configuration in a classification task when different lengths of a noise data sequence appear before useful signals. Examples of such different length are represented in Fig. 6(a) and Fig. 6(c ). The network was trained using the impulses as shown in Fig. 6(a). All testing samples with impulses like in Fig. 6(c) were misclassified. Fortunately, the real PD signal is supposed to be subjected to an impulse extracting algorithm. That algorithm keeps always almost the same noise data sequence before a useful signal impulse is extracted. If for some reason this condition is not met, then Power Spectral Density (PSD) of the recorded PD impulses can be used as an input of 1 D CNN [14]. Further, in the paper, this configuration is named as W0-S781-PSD.

To avoid overfitting in the configurations W0-S781PSD and W81-S781-PSD, a dropout mechanism was applied (dropout layers in the network structure), consisting of randomly dropping a neuron so that it would not contribute to the learning and predictive process. Founded network configuration that shows a good classification performance when PSD is used as an input, is represented in Tab. 7.

The result of classification of PD impulses, processed according to W0-S781 configuration, is presented in Fig. 7. For this configuration was used the network structure, shown in Fig. 5. From the confusion matrix, shown in Fig. 7(a), follows that in the case of class number 1 and class number 5 , we obtained a relatively high error rate. Class number 1 belongs to the experimental setup configuration with a distance between the electrodes $30 \mathrm{~mm}$, pressure in the vessel $300 \mathrm{kPa}$, and length of a protrusion $2 \mathrm{~mm}$. Three out of seventeen impulses were mistakenly classified as belonging to the configuration with a pressure in the vessel $250 \mathrm{kPa}$. The similar situation occurred in the configuration with a distance between the electrodes $40 \mathrm{~mm}$, pressure $300 \mathrm{kPa}$, and the protrusion 2 $\mathrm{mm}$ (class no5). In this case, $70 \%$ (14 out of 20 ) impulses were correctly classified as belonging to class no5, but five impulses were classified as belonging to class no6, and it is clearly not correct. Impulses in class no6 were obtained for the pressure in vessel $250 \mathrm{kPa}$. From this, it can be concluded, that pressures in the vessel may have a small impact on the impulse shape in the time domain and it leads to the high level of misclassification. On the contrary, pressures in the vessel in the case of the setup configuration with the $5 \mathrm{~mm}$ protrusion length, had a significant impact on the shape of the PD impulses, which

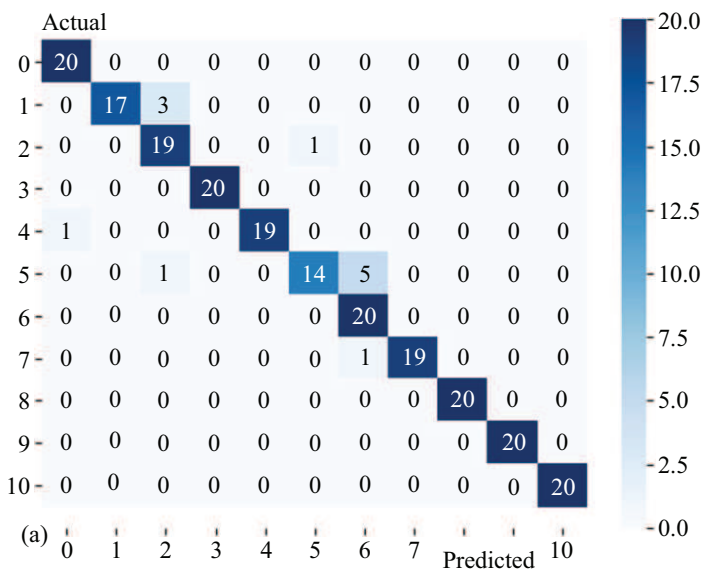

Fig. 7. Confusion matrix: (a) - of configuration W0-S781, and (b) - of configuration W81-S781

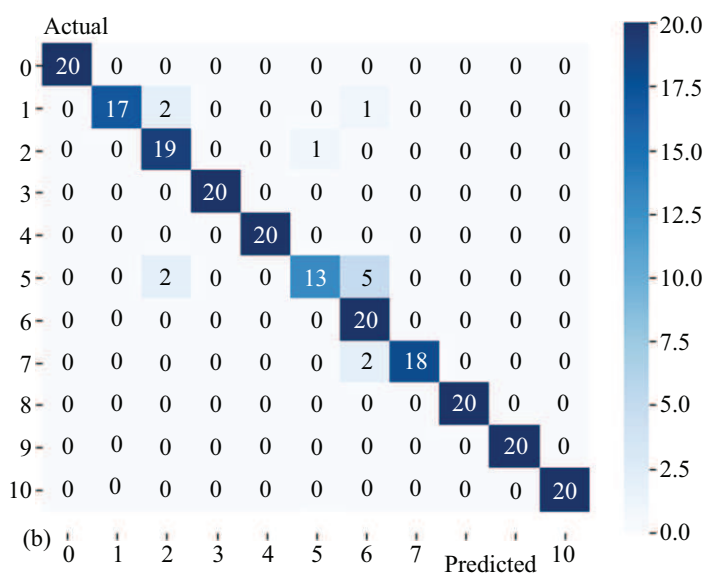

0.0 

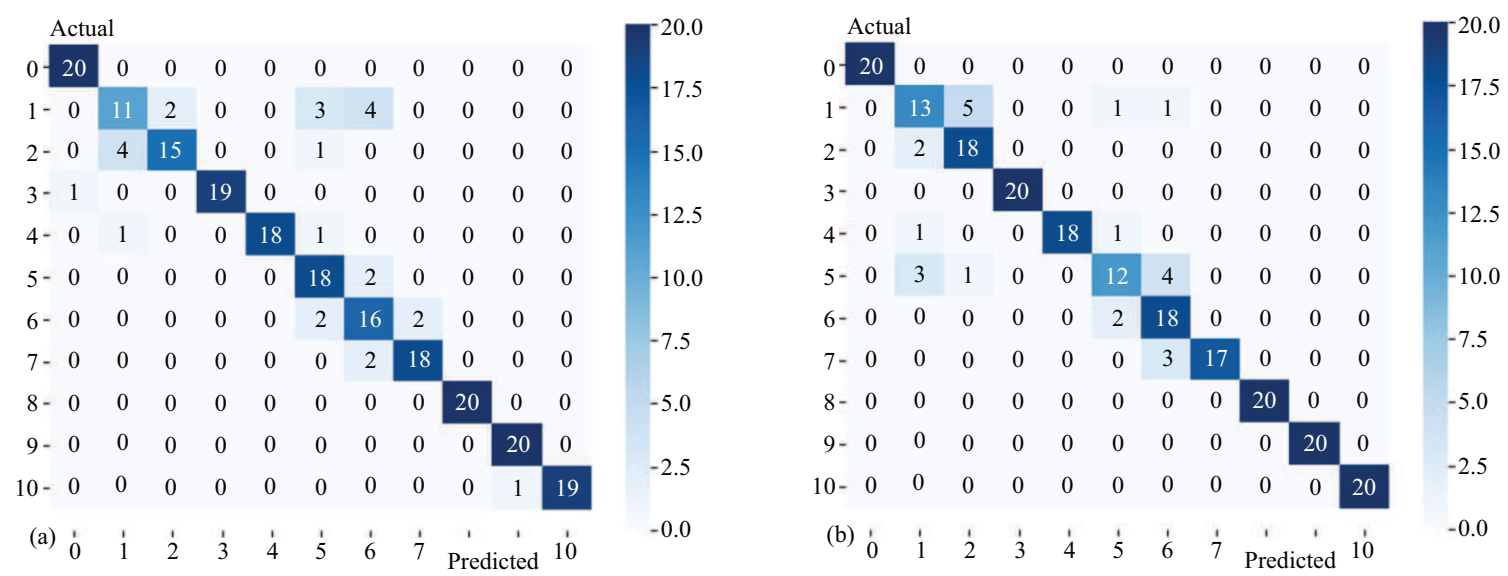

Fig. 8. Confusion matrix: (a) - of network configuration W0-S781-PSD, (b) - of configuration W81-S781-PSD

leads to classification accuracy from $95 \%$ (class numbers 4 and 7 ) to $100 \%$ (class no0 and no3). In Fig. 7(b) we represent the result of classification, when the filtered PD impulses were used, namely, for the configuration W81S781. It was found that there is no significant difference in classification performance between raw PD impulses and smoothed (filtered) PD impulses.

PD impulses for classes no8 and no9 were obtained from the internal discharge in a void. In the case of class number 8 , we used a void with a diameter $1.5 \mathrm{~mm}$ (Tab. 5). And in the case of class no9, it was a $2.5 \mathrm{~mm}$ diameter void. Classification algorithm showed $100 \%$ classification accuracy for this type of partial discharge signal for both filtered and unfiltered variants. PD impulses for classes number 10 were obtained from the surface discharge. Classification accuracy was $100 \%$ for this class for both filtered and unfiltered variants.

The result of the classification of PD impulses, processed according to W0-S781-PSD configuration, that is, using power spectral density, is presented in Fig. 8. For this configuration were used the network structures, shown in Tab. 7. It is evident that the overall classification accuracy is lower than in the case of using raw PD impulses, especially for class no1, 2, 5, 6, and 7. After applying of PD signal filtering, class no5 evinces the higher level of misclassifying than without filtering. The parameter that was changed to obtain this class is distance between the electrodes. Differentiability for class number 1 is remained on the same low level in both filtered and unfiltered variants. Worth mentioning that the number of misclassification events for configurations W0-S781-PSD and W81-S781-PSD never fell below 55\%. Therefore, the ability for a decision about what source of a PD it is remains.

\section{Conclusions}

Proposed one-dimensional convolutional neural network configurations can distinguish separate partial discharge impulses from different PD sources and different configurations of a source. In most cases, 20 out of 20 tested PD impulses within one class were classified correctly. A total of 11 classes were tested simultaneously. Misclassification events occurred in variants, in which pressures in the vessel were changed as a parameter. We can conclude from this that the pressure may have a small influence on the impulse shape. The effect is more pronounced in the case of using a power spectral density diagram as an input for the neural network. Such behavior of the classification algorithm can be explained by the PD impulses having similar features in the frequency domain, which causes difficulties for classification. Thus, it is recommended to use the time domain. It can be also concluded that there is no significant difference between the filtered and unfiltered PD signals in the case of using the proposed $1 \mathrm{D}$ CNN configurations. In addition, as a part of data preparation, thirteen variants with different sampling rate were generated and tested. Sampling rate $156 \mathrm{MS} / \mathrm{s}$ was found optimal for data processing by the proposed neural network configuration for both filtered and unfiltered signals. Variants with a sampling rate 39 $\mathrm{MS} / \mathrm{s}$ and $78 \mathrm{MS} / \mathrm{s}$ were found still useful, but the differentiability of a corona discharge significantly decreases in this case, especially for distinguishing between the variant obtained with different levels of pressure in the vessel. It is not possible to use a sampling rate less than 19 $\mathrm{MS} / \mathrm{s}$ due to signal aliasing occurrence. Thus, an optimal sampling rate for data acquisition can be accepted in the range 100-160 MS/s without significantly affecting the data analyzing performance.

\section{Acknowledgment}

This work was supported by the student research project SGS-2018-023.

\section{REFERENCES}

[1] M.-T. Nguyen, V.-H. Nguyen, S.-J. Yun, and Y.-H. Kim, "Recurrent Neural Network for Partial Discharge Diagnosis in Gas-Insulated Switchgear", Energies, vol. 11, no. 5, p. 1202, 2018. 
[2] H. H. Sinaga, T. R. Blackburn, and B. Phung, "Recognition of single and multiple partial discharge sources in transformers based on ultra-high frequency signals", IET Generation, Transmission \& Distribution, vol. 8, no. 1, pp. 160-169, 2014.

[3] M. Y. A. Khan and J.-Y. Koo, "Neural network-based diagnosis of partial discharge defects patterns at XLPE cable under DC stress", Electrical Engineering, vol. 99, no. 1, pp. 119-132, 2016.

[4] M. Quizhpi-Cuesta, F. Gomez-Juca, W. Orozco-Tupacyupanqui, and F. Quizhpi-Palomeque, "Classification of partial discharge in pin type insulators using fingerprints and neural networks", 2017 IEEE International Autumn Meeting on Power, Electronics and Computing (ROPEC), 2017.

[5] Schober and U. Schichler, "Application of Machine Learning for Partial Discharge Classification under DC Voltage", Proceedings of the Nordic Insulation Symposium, no. 26, pp. 16-21, 2019.

[6] W. J. K. Raymond, H. A. Illias, A. H. A. Bakar, and H. Mokhlis, "Partial discharge classifications: Review of recent progress", Measurement, vol. 68, pp. 164-181, 2015.

[7] B. Moons, D. Bankman, and M. Verhelst "', , Embedded Deep Learning, Springer, 2019.

[8] A. Kúchler, High Voltage Engineering: Fundamentals-Technology-Applications, Springer, 2018.

[9] D. Issa, M. F. Demirci, and A. Yazici, "Speech emotion recognition with deep convolutional neural networks", Biomedical Signal Processing and Control, vol. 59, p. 101894, 2020

[10] Li, X. Wang, X. Li, A. Yang, and M. Rong, "Partial Discharge Recognition with a Multi-Resolution Convolutional Neural Network", Sensors, vol. 18, no. 10, p. 3512, 2018.

[11] B. T. Phung, Z. Liu, T. R. Blackburn, and R. E. James, Recent Developments for On-line Partial Discharge Detection in Cables, School of Electrical Engineering and Telecommunications University of New South Wales, Australia, 2001.

[12] F. Chollet, Keras, 2015, https://github.com/fchollet/keras.

[13] Gibson and J. Patterson, Deep learning: a practitioner approach, O Reilly, 2017.
[14] L. Woon, Z. Aung, and A. El-Hag, "Intelligent Monitoring of Transformer Insulation Using Convolutional Neural Networks", Data Analytics for Renewable Energy Integration, Technologies, Systems and Society Lecture Notes in Computer Science, pp. 127-136, 2018

15] U. Fromm, "Interpretation of partial discharges at dc voltages", IEEE Transactions on Dielectrics and Electrical Insulation, vol. 2, no. 5, pp. 761-770, 1995.

16] V Peak signal detection in realtime time series data, https:/ /stackoverflow. com/questions/22583391/peak-signal-detection -in-realtime-xseries-data/54507329.

Received 22 October 2020

Mikhail Olkhovskiy is pursuing $\mathrm{PhD}$ degree from University of West Bohemia in Pilsen. He received his master degree in 2018 from the Faculty of Electrical Engineering.

Eva Müllerová received the master degree in Power Engineering from the VŠSE, Pilsen, Czech Republic, in 1998 and the $\mathrm{PhD}$ degree in Electrical Engineering from the University of West Bohemia, Pilsen, Czech Republic, in 1999. Her research includes the diagnostics of high voltage equipment, the investigation of degradation processes of insulating materials used in high voltage equipment, measuring and analysis of the discharges in insulating system. The main focus of research deals with the partial discharges and analysis acoustic responses in power transformer under $\mathrm{AC}$ and impulse voltages.

Petr Martínek obtained $\mathrm{PhD}$ degree in power engineering from University of West Bohemia, Pilsen in 2005. He received his master degree in 1999. Currently working at the Department of Power Engineering. His research interests include high voltage equipment testing and diagnostics, partial discharge analysis, electromagnetic compatibility. 